\title{
Proliferation of Keratinocytes Induced by Adipose- Derived Stem Cells on a Chitosan Scaffold and Its Role in Wound Healing, a Review
}

\author{
Sankaralakshmi Gomathysankar ${ }^{1}$, Ahmad Sukari Halim ${ }^{1}$, Nik Soriani Yaacob ${ }^{2}$ \\ ${ }^{1}$ Reconstructive Sciences Unit, ${ }^{2}$ Department of Chemical Pathology, School of Medical Sciences, Universiti Sains Malaysia, Kelantan, Malaysia
}

In the field of tissue engineering and reconstruction, the development of efficient biomaterial is in high demand to achieve uncomplicated wound healing. Chronic wounds and excessive scarring are the major complications of tissue repair and, as this inadequate healing continues to increase, novel therapies and treatments for dysfunctional skin repair and reconstruction are important. This paper reviews the various aspects of the complications related to wound healing and focuses on chitosan because of its unique function in accelerating wound healing. The proliferation of keratinocytes is essential for wound closure, and adipose-derived stem cells play a significant role in wound healing. Thus, chitosan in combination with keratinocytes and adipose-derived stem cells may act as a vehicle for delivering cells, which would increase the proliferation of keratinocytes and help complete recovery from injuries.

Keywords Chitosan / Keratinocytes / Stem cells / Wound healing

\author{
Correspondence: \\ Ahmad Sukari Halim \\ Reconstructive Sciences Unit, School \\ of Medical Sciences, Universiti Sains \\ Malaysia, 16150 Kubang Kerian, \\ Kelantan, Malaysia \\ Tel: $+60-09-7666000$ \\ Fax: $+60-09-7656434$ \\ E-mail: ashalim@usm.my
}

\begin{abstract}
The work related to this paper was supported by a Research University Grant (1001/PPSP/813058) from Universiti Sains Malaysia.
\end{abstract}

No potential conflict of interest relevant to this article was reported.

Received: 20 Mar $2014 \bullet$ Revised: 25 Apr $2014 \bullet$ Accepted: 26 Apr 2014

pISSN: 2234-6163 • elSSN: 2234-6171 • http://dx.doi.org/10.5999/aps.2014.41.5.452 • Arch Plast Surg 2014;41:452-457

\section{INTRODUCTION}

The skin is the protective, defensive barrier from the outside world; therefore, any break or injury in the skin should be efficiently mended. Wound-healing biology involves the signaling pathways that trigger relatively inactive cell lineages to the wound margin for the proliferation, invasion and reconstruction of new matrices in the wound gap [1]. Studies have established that the incidence of severe burns in the United States is estimated to be 70,000 per year [2]; the occurrence of venous leg ulcer is between 600,000 and 1,500,000 [3]; and the prevalence of chronic foot wounds in people suffering from diabetes is 15 to 20 percent [4]. Thus, the dressing cost alone for the above-mentioned cases has been calculated as $\$ 5$ billion per year [5]. Although multiple treatments such as skin grafting and skin reconstruction with biomaterials exist, further development and refinement of skin substitutes or biomaterials are essential for perfect skin reconstruction.

\section{WOUND-HEALING PROCESS}

Wound healing is a complex mechanism that begins with an inflammatory phase followed by re-epithelialization and ending with a remodeling phase. Tissue injury disrupts vascular vessels and initiates extravasation. The inflammatory response begins with the release of growth factors, cytokines and components of extracellular matrices (ECM). Then, the tissue re-epithelialization phase, which is a process mainly involving the migration 
and proliferation of keratinocytes to resurface the wound area with a layer of new epithelium, takes place. As this epidermal layer continues migrating, the keratinocytes at the wound margin begin to proliferate and migrate to contact the wound margin $[1,6-8]$. Finally, the remodeling phase, where the collagen fibers reorganize and mature to gain tensile strength, occurs [8]. Thus, the wound-healing mechanism is a complex chain of events involving different cell-to-cell interactions and interactions among tissues that are impaired due to a number of medical conditions. Skin injuries could be healed more quickly, but re-epithelialization is not always perfect and leaves a connective tissue scar. Therefore, the study of the proliferation of keratinocytes, which plays a major role in re-epithelialization, is important for tissue reconstruction.

\section{SKIN SUBSTITUTES FOR WOUND HEALING}

The initial treatments for severe injuries and burn cases include autografts, allografts and xenografts. However, these treatments often have limited donor sites. Recently, studies have shown that bioengineered skin substitutes are an advancement in tissue engineering with a wide range of applications in wound healing [5]. Based on the depth of the injuries, wounds can be classified into epidermal, superficial partial thickness, deep partial thickness and full thickness [9]. Therapeutic approaches for the treatment of deep dermal and full-thickness injuries remain unsatisfactory; therefore, more effective treatment strategies are needed [10].

In cases of severe burn injuries, a stretched, meshed skin graft could be used. The focus has then moved to skin substitutes, and the main goal behind this method is to accelerate wound healing using the normal repair mechanism, provide a surface for cells to proliferate and prevent bacterial infection. Based on the requirements of skin injury, different types of skin substitutes are used for specific purposes [11-20]. Table 1 shows some of the widely used skin substitutes $[18,19]$.

These bioengineered skin substitutes can offer four functions such as protection: creating a defense barrier to micro organisms, procrastination: to achieve permanent wound closure particularly in case of extensive burn injuries, promotion: delivering matrix components, growth factors and cytokines, provision: incorporation of dermal collagen or cultured cells at the wound site [21]. Although the meshed skin graft covers a greater area, most of the skin grafts and skin substitutes that has been used have various disadvantages, such as slow epithelialization, delayed wound healing, graft contraction, scarring of tissues, slow vascularisation and inadequate acceleration of wound healing [22].

Additionally, skin substitutes should be cost effective, readily available, resistant to infection and have a longer shelf life. Unfortunately skin substitutes with all of these properties are unavailable in the market. Because of the great importance and high demand of skin substitute products, research should be carried out to develop an ideal skin substitute [18].

\section{CHITOSAN IN WOUND HEALING}

Chitosan is an abundantly available biopolymer composed of (1-4)-2-acetamido-2-deoxy-b-D-glucan (N-acetyl D-glucosamine) and (1-4)-2-amino-2-deoxyb-D-glucan (D-glucosamine) units, which are partially derived from the deacetylation of chitin polymers [23]. Chitin is most commonly found in invertebrates such as crustaceans or insect cuticles, mushrooms, green algae, yeasts and the shells of shrimp and crab [24-27]. However, the poor solubility of chitin limits its practical usage. The presence of amino groups differentiates chitosan from chitin and gives unique properties to the chitosan polymer, which has more clinical and non-clinical applications (Fig. 1) [28,29]. Table 1. Various skin substitutes that have been used in recent years $[18,19]$

\begin{tabular}{|ll}
\hline Skin substitutes & \multicolumn{1}{c}{ Types } \\
\hline Temporary impervious dressing materials & 1. Single-layer materials \\
& a) Biological dressing substitute (e.g., amniotic membrane) \\
& b) Synthetic dressing substitute (e.g., Synthetic polymer sheet, polymer foam or spray) \\
& 2. Bi-layered tissue engineered materials (e.g., TransCyte) \\
Single-layer durable skin substitutes & 1. Epidermal substitutes (e.g., Cultured epithelial autograft, Apligraft) \\
& 2. Dermal substitutes (e.g., Bovine and porcine collagen sheet, bovine and human dermal matrix) \\
Composite skin substitutes & 1. Skin graft \\
& a) Allograft \\
& b) Xenograft \\
2. Tissue-engineered skin & a) Dermal regeneration template (e.g., Integra) \\
biological skin substitutes & b) Biobrane \\
Synthetic skin substitutes & Skin allograft, xenograft and amnion, cultured epithelial autografts, extracellular matrix \\
\hline
\end{tabular}




\section{Fig. 1. Chitosan derived from deacetylation of chitin}

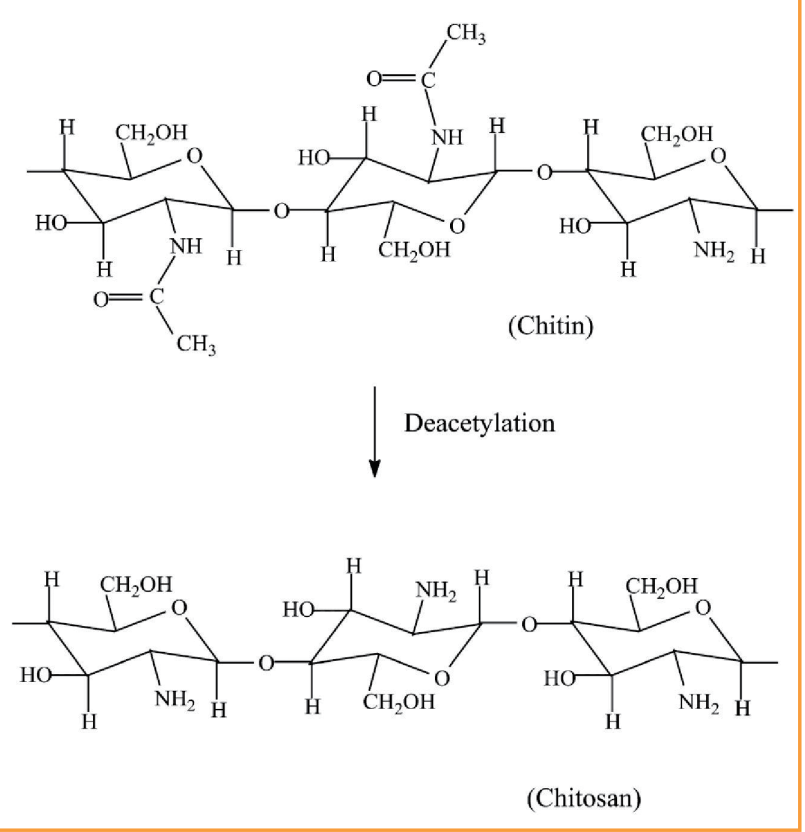

Chitosan biopolymers have been widely used in the fields of biotechnology, cosmetics, biomedicine, food and agriculture [26]. In tissue engineering, chitosan that accelerates wound healing has been used for wound dressings and creating artificial skin [27]. Chitosan is found to be biodegradable and biocompatible and is an excellent hemostatic and analgesic agent with antioxidant properties [26]. Chitosan enhances the functions of inflammatory cells and growth factors, thereby promoting granulation and remodeling of damaged tissues in large, open wounds of animals [30]. It has been shown that chitosan hydrogel interacts with fibroblast growth factor (FGF-2) on an openwound surface in a mouse model. This interaction resulted in contraction of the wound, formation of granulation tissue, closure and healing of the wound [31]. Recent studies have shown that a bilayer chitosan membrane, which consists of an upper chitosan film layer attached to an inner layer of porous membrane, serves as an efficient skin-regenerating template for treating third-degree burns and cutaneous wounds. This chitosan bilayer has the potential to enhance the proliferation of fibroblasts, thereby forming a monolayer to cover the wound surface [32]. The three dimensional structural organization of chitosan is essential to serve as a vehicle for delivering and retaining the cells at a specific site and to initiate appropriate cell-to-cell interactions [33]. Chitosan supports the adhesion and activation of platelets, which are enhanced by plasma and extracellular matrix proteins [34]. Based on the literature, tissue-engineering scaffolds should 1) be biodegradable to favor the cured tissue in replacing the biomaterial, 2) not trigger the acute or chronic inflammatory responses, 3) have surface properties that enhance the attachment, proliferation and differentiation of cells, 4) mimic the skin in vitro, 5) have suitable mechanical properties, and 6) be suitable for manufacturing into different shapes [28]. Chitosan when compared with the above mentioned skin substitutes, has all of these remarkable properties which make chitosan scaffold as a promising future in the management of wound healing. Also, its availability in different forms would serve as efficient scaffolds in the treatment of acute and chronic wound injuries.

\section{KERATINOCYTES AND CHITOSAN}

Keratinocytes are the predominant cell components of the epidermis. These cells play a significant role in the wound-healing process because they are involved in the complex mechanisms of initiation, proliferation and re-epithelialization of wound healing. Normal and healthy keratinocytes differ from the keratinocytes at the non-healing chronic wound edges. In cases of injuries, the migration of basal keratinocytes from the wound margin and cut epidermal appendages to the denuded wound surface are essential to carry forward or move over the newly reconstructed dermal scaffolding. The stratified keratinocytes proliferate and differentiate to produce neoepidermis, which covers the entire wound surface and restores the skin function [35]. For the successful closure of wounds, the proliferation of keratinocytes is essential to facilitate communication with other cell types that are involved in wound healing [36].

Because chitosan has been employed as the best biomaterial for wound dressing, studies have been conducted to determine the interactions between chitosan and keratinocytes. The degree of acetylation (DA) is a term used to define chitin and chitosan. DA is an essential structural parameter that influences biological and wound-healing properties [37-39]. Cultures of keratinocytes were analyzed on five different chitosan films with DAs ranging from $2.5 \%$ to $47 \%$, and the cell adhesion and proliferation of the keratinocytes on these chitosan films were investigated. The DA does not influence the cytocompatibility of chitosan films with keratinocytes in vitro; however, fully deacetylated chitosan films allowed for better adhesion to keratinocytes, resulting in their better proliferation. This finding indicates that chitosan films with low DA could act as efficient biomaterials because they would adhere to fibroblasts and induce the proliferation of keratinocytes and re-epithelialization [40].

The effects of chitin and chitosan on the proliferation of keratinocytes in vitro were studied. Primary human keratinocytes and an immortalized human keratinocyte cell line ( $\mathrm{HaCaT})$ were cultured with and without an irradiated fibroblast feeder layer. Chitosan and the primary keratinocytes with the irradiat- 
ed feeder layer supported the growth and proliferation of keratinocytes in vitro [41]. This study proves that highly deacetylated chitosan has more potential for wound healing. However, the mechanism of interaction between chitosan and keratinocytes is not clear [42]. Hence it is important to study the proliferation of keratinocytes on chitosan scaffold.

\section{ADIPOSE-DERIVED STEM CELLS AND CHITOSAN}

Current research findings reveal that bone marrow-derived stem cells (BMSCs) were found to play an important role in tissue repair. BMSCs secrete growth factors that enhance the stimulation, proliferation and regeneration of damaged cells [43]. The transplantation of BMSCs require harvesting of large numbers of bone marrow cells under general anesthesia, which leads to several complications that limit its use [44,45]. Adipose-derived stem cells (ASCs) are pluripotent stem cells that are derived from adipose tissue and have characteristics similar to mesenchymal stem cells derived from bone marrow. ASCs were found to have potent applications in the repair and regeneration of damaged tissues by helping in wound healing and in treatment for scarring and photoaging. Therefore, ASCs can be used therapeutically to treat chronic wounds and other conditions. However, for massive tissue damage, the available ASCs are insufficient to efficiently repair the damaged tissue, but this could be overcome by using adipose tissue obtained from liposuction procedures [46,47]. These ASCs, when combined with chitosan, have been found to increase the repairing and healing potential of damaged tissues. The microenvironment for skin regeneration mainly depends on interactions between stem cell progenitors and their niche [48]; therefore, any tissue-engineered reconstruct should provide a suitable microenvironment for the cells to proliferate and differentiate.

ASCs were seeded on silk fibroin chitosan (SFCS), and the impact of ASC-SFCS on wound healing was evaluated. Because of its biocompatible nature, silk fibroin was hybridized to chitosan, and the resulting hybrid matrix mimicked the constituents of the extracellular matrix and served as a substrate for cell adhesion and migration and the incorporation of tissues $[49,50]$. In a murine cutaneous injury model, ASC engrafts proliferated and differentiated into vascular, fibroblastic and epithelial cell phenotypes in their newly established microenvironment. The ASCs-SFCS also showed vascular enhancement, and SFCS acted as a delivery vehicle that provided a supportive niche for the migration, proliferation and differentiation of the cells. This study elucidated that ASC-SFCS supports the engraftment of stem cells and their differentiation into epithelial and fibrovas- cular components; therefore, it could be applied in the clinical setting [51]. It has also been found that ASCs induce osteogenic and chondrogenic differentiation in chitosan-agglomerated scaffolds, which are used as substitutes for the ECM [52]. In a similar study, human Hair Follicle Stem Cells (HFSCs) combined with fibroblasts were seeded in chitosan and found to be successful in accelerating wound healing in full-thickness wounds of irradiated rats [53]. Studies also revealed that ASCs could enhance the proliferation of $\mathrm{HaCat}$ cells (immortalized human keratinocytes) and accelerate in vitro wound healing [54]. Therefore, it is critical to study the proliferation of human keratinocytes that is enhanced by ASCs on a chitosan scaffold in vitro.

\section{CONCLUSIONS}

The unique properties of chitosan may increase the proliferation of keratinocytes when seeded with ASCs. It is therefore important to study the proliferation of human keratinocytes induced by ASCs using the in vitro model of the chitosan scaffold. This scaffold could help maximize the healing potential, for proper skin regeneration. Research should be carried out to establish the stimulatory effects of ASCs on a chitosan scaffold and to determine the cellular and molecular mechanisms involved. Furthermore, importance should be placed on the efficient development of chitosan skin substitutes with long-term safety and longer shelf life. Thus, this review has highlighted the importance of chitosan in tissue reconstruction, which paves the way for novel therapeutic strategies.

\section{REFERENCES}

1. Kirsner RS, Eaglstein WH. The wound healing process. Dermatol Clin 1993;11:629-40.

2. Boyce ST, Warden GD. Principles and practices for treatment of cutaneous wounds with cultured skin substitutes. Am J Surg 2002; 183:445-56.

3. Phillips TJ. Current approaches to venous ulcers and compression. Dermatol Surg 2001;27:611-21.

4. Reiber GE, Boyko EJ, Smith DG. Lower extremity foot ulcers and amputations in diabetes. In: Harris MI, Cowie CC, Stern MP, editors. Diabetes in America. 2nd ed. Washington, DC: U.S. Government Printing Office; 1995. p.409-28.

5. Eisenbud D, Huang NF, Luke S, et al. Review skin substitutes and wound healing: current status and challenges. Wounds 2004;16:2-17.

6. Werner S, Grose R. Regulation of wound healing by growth factors and cytokines. Physiol Rev 2003;83:835-70.

7. Rappolee DA, Patel Y, Jacobson K. Expression of fibroblast 
growth factor receptors in peri-implantation mouse embryos. Mol Reprod Dev 1998;51:254-64.

8. Martin P. Wound healing: aiming for perfect skin regeneration. Science 1997;276:75-81.

9. Papini R. Management of burn injuries of various depths. BMJ 2004;329:158-60.

10. Steeper R. A critical review of the aetiology of diabetic neuropathic ulcers. J Wound Care 2005;14:101-3.

11. Braddock M, Campbell CJ, Zuder D. Current therapies for wound healing: electrical stimulation, biological therapeutics, and the potential for gene therapy. Int J Dermatol 1999; 38:808-17.

12. Atiyeh BS, Hayek SN, Gunn SW. New technologies for burn wound closure and healing-review of the literature. Burns 2005;31:944-56.

13. Horch RE, Kopp J, Kneser U, et al. Tissue engineering of cultured skin substitutes. J Cell Mol Med 2005;9:592-608.

14. Atiyeh BS, Costagliola M. Cultured epithelial autograft (CEA) in burn treatment: three decades later. Burns 2007; 33:405-13.

15. Clark RA, Ghosh K, Tonnesen MG. Tissue engineering for cutaneous wounds. J Invest Dermatol 2007;127:1018-29.

16. MacNeil S. Progress and opportunities for tissue-engineered skin. Nature 2007;445:874-80.

17. Patel M, Fisher JP. Biomaterial scaffolds in pediatric tissue engineering. Pediatr Res 2008;63:497-501.

18. Halim AS, Khoo TL, Mohd Yussof SJ. Biologic and synthetic skin substitutes: An overview. Indian J Plast Surg 2010;43: S23-8.

19. Kumar P. Classification of skin substitutes. Burns 2008;34: 148-9.

20. Jones I, Currie L, Martin R. A guide to biological skin substitutes. Br J Plast Surg 2002;55:185-93.

21. Shakespeare PG. The role of skin substitutes in the treatment of burn injuries. Clin Dermatol 2005;23:413-8.

22. Shevchenko RV, James SL, James SE. A review of tissue-engineered skin bioconstructs available for skin reconstruction.J R Soc Interface 2010;7:229-58.

23. Rinaudo M. Chitin and chitosan: properties and applications. Prog Polym Sci 2006;31:603-32.

24. Rane KD, Hoover DG. Production of chitosan by fungi. Food Biotechnol 1993;7:11-33.

25. Aranaz I, Harris R, Heras A. Chitosan amphiphilic derivatives. Chemistry and applications. Curr Org Chem 2010;14: 308-30.

26. Aranaz I, Mengíbar M, Harris R, et al. Functional characterization of chitin and chitosan. Curr Chem Biol 2009;3:20330.
27. Zhang J, Xia W, Liu P, et al. Chitosan modification and pharmaceutical/biomedical applications. Mar Drugs 2010;8: 1962-87.

28. Croisier F, Jerome C. Chitosan-based biomaterials for tissue engineering. Eur Polym J 2013;49:780-92.

29. Prado AG, Torres JD, Faria EA, et al. Comparative adsorption studies of indigo carmine dye on chitin and chitosan. J Colloid Interface Sci 2004;277:43-7.

30. Ueno H, Mori T, Fujinaga T. Topical formulations and wound healing applications of chitosan. Adv Drug Deliv Rev 2001;52:105-15.

31. Obara K, Ishihara M, Fujita M, et al. Acceleration of wound healing in healing-impaired $\mathrm{db} / \mathrm{db}$ mice with a photocrosslinkable chitosan hydrogel containing fibroblast growth factor-2. Wound Repair Regen 2005;13:390-7.

32. Mao JS, Zhao LG, Yin YJ, et al. Structure and properties of bilayer chitosan-gelatin scaffolds. Biomaterials 2003;24: 1067-74.

33. Liao F, Chen Y, Li Z, et al. A novel bioactive three-dimensional beta-tricalcium phosphate/chitosan scaffold for periodontal tissue engineering. J Mater Sci Mater Med 2010;21: 489-96.

34. Lord MS, Cheng B, McCarthy SJ, et al. The modulation of platelet adhesion and activation by chitosan through plasma and extracellular matrix proteins. Biomaterials 2011;32: 6655-62.

35. Singer AJ, Clark RA. Cutaneous wound healing. N Engl J Med 1999;341:738-46.

36. Pastar I, Stojadinovic O, Tomic-Canic M. Role of keratinocytes in healing of chronic wounds. Surg Technol Int 2008; 17:105-12.

37. Tomihata K, Ikada Y. In vitro and in vivo degradation of films of chitin and its deacetylated derivatives. Biomaterials 1997;18:567-75.

38. Varum KM, Myhr MM, Hjerde RJ, et al. In vitro degradation rates of partially $\mathrm{N}$-acetylated chitosans in human serum. Carbohydr Res 1997;299:99-101.

39. Sathirakul K, How NC, Stevens WF, et al. Application of chitin and chitosan bandages for wound healing. Adv Chitin Sci 1996;1:490-2.

40. Chatelet C, Damour O, Domard A. Influence of the degree of acetylation on some biological properties of chitosan films. Biomaterials 2001;22:261-8.

41. Rheinwald JG, Green H. Serial cultivation of strains of human epidermal keratinocytes: the formation of keratinizing colonies from single cells. Cell 1975;6:331-43.

42. Howling GI, Dettmar PW, Goddard PA, et al. The effect of chitin and chitosan on the proliferation of human skin fibro- 
blasts and keratinocytes in vitro. Biomaterials 2001;22: 2959-66.

43. Phinney DG, Prockop DJ. Concise review: mesenchymal stem/multipotent stromal cells: the state of transdifferentiation and modes of tissue repair--current views. Stem Cells 2007;25:2896-902.

44. Chen L, Tredget EE, Wu PY, et al. Paracrine factors of mesenchymal stem cells recruit macrophages and endothelial lineage cells and enhance wound healing. PLoS One 2008; 3:e1886.

45. Wu Y, Chen L, Scott PG, et al. Mesenchymal stem cells enhance wound healing through differentiation and angiogenesis. Stem Cells 2007;25:2648-59.

46. Gimble JM, Katz AJ, Bunnell BA. Adipose-derived stem cells for regenerative medicine. Circ Res 2007;100:1249-60.

47. Zuk PA, Zhu M, Mizuno H, et al. Multilineage cells from human adipose tissue: implications for cell-based therapies. Tissue Eng 2001;7:211-28.

48. Wong VW, Levi B, Rajadas J, et al. Stem cell niches for skin regeneration. Int J Biomater 2012;2012:926059.
49. Gobin AS, Butler CE, Mathur AB. Repair and regeneration of the abdominal wall musculofascial defect using silk fibroin-chitosan blend. Tissue Eng 2006;12:3383-94.

50. Khor E, Lim LY. Implantable applications of chitin and chitosan. Biomaterials 2003;24:2339-49.

51. Altman AM, Yan Y, Matthias N, et al. IFATS collection: $\mathrm{Hu}-$ man adipose-derived stem cells seeded on a silk fibroin-chitosan scaffold enhance wound repair in a murine soft tissue injury model. Stem Cells 2009;27:250-8.

52. Malafaya PB, Pedro AJ, Peterbauer A, et al. Chitosan particles agglomerated scaffolds for cartilage and osteochondral tissue engineering approaches with adipose tissue derived stem cells. J Mater Sci Mater Med 2005;16:1077-85.

53. Lee SH, Jin SY, Song JS, et al. Paracrine effects of adiposederived stem cells on keratinocytes and dermal fibroblasts. Ann Dermatol 2012;24:136-43.

54. Mohd Hilmi AB, Halim AS, Jaafar $\mathrm{H}$, et al. Chitosan dermal substitute and chitosan skin substitute contribute to accelerated full-thickness wound healing in irradiated rats. Biomed Res Int 2013;2013:795458. 